\title{
New Results on Solar Neutrinos
}

\author{
A. Bellerive ${ }^{* \dagger}$ \\ Ottawa-Carleton Institute for Physics \\ Department of Physics \\ Carleton University \\ 1125 Colonel By Drive, Ottawa, K1S 5B6, Canada \\ E-mail: alain_bellerive@carleton.ca
}

This paper reviews the constraints on the solar neutrino mixing parameters with data collected by the Homestake, SAGE, GALLEX, Kamiokande, SuperKamiokande, Borexino and SNO experiments. An emphasis will be given to the global solar neutrino analyses in terms of matterenhanced oscillation of two and three active flavors. The results to-date, including both solar model dependent and independent measurements, indicate that electron neutrinos are changing to other active types on route to the Earth from the Sun. The total flux of solar neutrinos is found to be in very good agreement with solar model calculations. Today, solar neutrino measurements focus on greater accuracy for mixing parameters and on better sensitivity to low neutrino energies. This article also summarizes near future prospects in the field of solar neutrino physics.

35th International Conference of High Energy Physics - ICHEP2010,

July 22-28, 2010

Paris France

\footnotetext{
* Speaker.

${ }^{\dagger}$ On behalf of the SNO Collaboration.
} 


\section{Introduction}

The deficit of neutrinos detected coming from the Sun compared with our expectations based on laboratory measurements, known as the Solar Neutrino Problem, has remained one of the outstanding problems in basic physics for over thirty years. It appeared inescapable that either our understanding of the energy producing processes in the Sun is seriously defective, or neutrinos, some of the fundamental particles in the Standard Model, have important properties which have yet to be identified. It was indeed argued by some that we needed to change our ideas on how energy was produced in fusion reactions inside the Sun. Others suggested that the problem arose due to peculiar characteristics of neutrinos such as oscillations and matter effects. It is then useful to review the evolution of our understanding from the data collected by various solar neutrino experiments. For completeness, near term prospects are included in the discussion presented here.

\section{Solar Neutrinos}

The detailed prediction of the electron neutrino flux created by the thermonuclear reactions in the interior of the Sun was performed by John Bahcall and his collaborators (for example see [1]). Their calculations are referred to as the Standard Solar Model (SSM). In this paper, the SSM calculations are used to compare experimental results and theoretical predictions. The relevant point for the discussion that follows is that SSM theoretical uncertainties on many solar fluxes have been reduced [2]. As a result, the errors on the ${ }^{7} \mathrm{Be}, \mathrm{pep},{ }^{8} \mathrm{~B}$ and hep fluxes are now $\pm 6 \%, \pm 1.1 \%$, $\pm 11 \%$ and $\pm 16 \%$, respectively.

\section{Chlorine Experiment}

The exploration of solar neutrinos started in the mid-1960's with Ray Davis [3]. It led to the first experiment that successfully detected neutrinos coming from the Sun. The experiment of Davis and his team was carried out deep underground in the Homestake mine in the US. The detector was based on a concept first proposed by Bruno Pontecorvo at Chalk River in 1946, in which neutrino reactions on chlorine are measured. Neutrinos striking chlorine can make an isotope of argon through the reaction

$$
v_{e}+{ }^{37} \mathrm{Cl} \rightarrow e^{-}+{ }^{37} \mathrm{Ar},
$$

with an energy threshold of $0.814 \mathrm{MeV}$. The first results were announced in 1968.

The chlorine experiment took data until 1995 and clearly showed argon atoms produced by neutrinos, but the predicted production rate was four times the measured value [4]:

$$
\Phi_{\mathrm{Cl}}=2.56 \pm 0.23 \mathrm{SNU}
$$

while the SSM predicted rate was about $\Phi_{\mathrm{Cl}}(\mathrm{SSM})=7.6 \mathrm{SNU}$. A SNU (Solar Neutrino Unit) is the product of the solar neutrino fluxes (measured or calculated) and the calculated cross sections. Hence one SNU equals one capture per second and per $10^{36}$ target atoms. 


\section{Gallium Experiments}

While the chlorine detector was mainly sensitive to the highest energy neutrinos, two gallium experiments, one at the Baksan laboratory [5] in Russia and one at the Gran Sasso laboratory [6] in Italy, were set up to test the oscillation hypothesis at lower energy. Like the ${ }^{37} \mathrm{Cl}$ detector, the gallium detectors could only detect electron type neutrinos because they looked for the reaction

$$
v_{e}+{ }^{71} G a \rightarrow e^{-}+{ }^{71} G e .
$$

The energy threshold of the ${ }^{71} \mathrm{Ga}$ detectors is $0.233 \mathrm{MeV}$ and hence allows the interaction of $p p$, ${ }^{7} \mathrm{Be},{ }^{8} \mathrm{~B}$, and pep neutrinos. The Russian-American group (SAGE) used a liquid metal target which contained 50 tons of gallium; while the European group (GALLEX/GNO) used 30 tons of natural gallium in an aqueous acid solution. Small proportional counters are used to count the germanium from the radiochemical target. The ${ }^{71} \mathrm{Ge}$ electron capture decay occurs with a mean-life of 16.5 days. The Auger electrons and X-rays produce the typical L-peak and K-peak energy distribution. As a cross-check, both peaks are counted separately. Both experiments found about half of the expected rate. The most recent results of SAGE and GALLEX/GNO yield [7]

$$
\Phi_{\mathrm{Ga}}=66.1 \pm 3.1 \mathrm{SNU} .
$$

The data are incompatible with the SSM since the expected rate is about $\Phi_{\mathrm{Ga}}(\mathrm{SSM})=129 \mathrm{SNU}$.

\section{Kamiokande and SuperKamiokande}

Following the first observations from the $\mathrm{Cl}$ experiment the first priority was obviously an experimental confirmation of the solar-neutrino deficit. This was provided in 1987 by the Kamiokande water Čerenkov detector [8] in Japan, which also saw a significant (but, interestingly enough, not an identical) suppression of the measured rate of neutrinos from the Sun. The Kamiokande Collaboration demonstrated that the neutrinos are actually coming from the direction of the Sun by reconstructing the direction of flight of the incident neutrinos from the neutrino-electron scattering (ES) reaction $v_{x}+e^{-} \rightarrow v_{x}+e^{-}$. Light water detectors are mainly sensitive to $v_{e}$, but also to $v_{\mu}$ and $v_{\tau}$, such that $\sigma\left(v_{\mu \tau} e^{-} \rightarrow v_{\mu \tau} e^{-}\right) \simeq 0.15 \times \sigma\left(v_{e} e^{-} \rightarrow v_{e} e^{-}\right)$.

The follow-up of the Kamiokande project is called the SuperKamiokande (SK) experiment [9]. It was built to investigate in more detail the nature of atmospheric and solar neutrino oscillations. The SK detector is a huge, $40 \mathrm{~m}$ in diameter and $40 \mathrm{~m}$ high, circular cylinder filled with 50,000 tons of ultra-pure light water. The SK detector operates at an energy threshold that permits the study of the ${ }^{8} B$ neutrinos. It is divided into an outer detector to veto incoming cosmic ray muons and to shield external low energy background; and an inner detector (32,000 tons, of which 22,500 tons is the active fiducial volume) viewed by 11,146 PMT. As in Kamiokande, solar neutrinos are observed by detecting Čerenkov photons emitted by the electrons resulting from ES events. The event rate was about 15 events per day (substantially larger than the rate in the radiochemical experiments). The SK-I data allows measurements of the time dependence of the ES rate. It led to the measurement of the day/night rate asymmetry [9]

$$
A_{\mathrm{DN}}=2 \frac{\Phi_{\mathrm{D}}-\Phi_{\mathrm{N}}}{\Phi_{\mathrm{D}}+\Phi_{\mathrm{N}}}=-0.021 \pm 0.020_{-0.012}^{+0.013},
$$


and the precise determination of the ES neutrino rate $\Phi_{\mathrm{ES}}=(2.35 \pm 0.02 \pm 0.08) \times 10^{6} \mathrm{~cm}^{-2} \mathrm{~s}^{-1}[9]$. The energy shape of the recoil electron agrees well, within experimental errors, with that predicted from the neutrino spectrum from the beta decay of ${ }^{8} B$. The measurement of the absolute flux, however, is about $41 \%$ of that predicted by the SSM.

The results of the second phase of SK (SK-II) are consistent with the results of SK-I. The measured ES neutrino rate is $\Phi_{\mathrm{ES}}=2.38 \pm 0.05_{-0.15}^{+0.16} \times 10^{6} \mathrm{~cm}^{-2} \mathrm{~s}^{-1}$ [10]; while the day-night difference is found to be $A_{\mathrm{DN}}=-0.063 \pm 0.042 \pm 0.037$ [10].

\section{Borexino}

The Borexino detector [11] is located in Hall C of the Gran Sasso Laboratory. It is a 300 ton liquid-scintillator based detector with 100 tons of active fiducial mass in a $8.3 \mathrm{~m}$ diameter spherical nylon bag surrounded by a 2.6 meter thick spherical shell filled with buffer oil. The liquid scintillator and buffer liquid are viewed by 2,240 PMT which are mounted inside a $13.5 \mathrm{~m}$ diameter stainless steel tank; which is in turn surrounded by a $18 \mathrm{~m}$ spherical tank filled with ultra-pure light water to act as a radiation shield.

Solar neutrinos are detected in Borexino through their elastic scattering on electrons in the scintillator. Electron neutrinos $\left(v_{e}\right)$ interact through charged and neutral currents and in the energy range of interest have a cross section $\sim 5$ times larger than $v_{\mu}$ and $v_{\tau}$, which interact only via the neutral current. The electrons scattered by neutrinos are detected by means of the scintillation light. Borexino is the first solar neutrino experiment to report a real-time observation of low energy ${ }^{7} \mathrm{Be}$ neutrinos. The signature for the mono-energetic ${ }^{7} \mathrm{Be}$ neutrinos in Borexino is the Compton-like edge of the recoil electrons at $665 \mathrm{keV}$.

Borexino reported the direct measurement of the $0.862 \mathrm{MeV}{ }^{7} \mathrm{Be}$ solar neutrinos with the Borexino detector from an analysis of 192 live days in the period from May 16, 2007 to April 12, 2008 , totaling a 41.3 ton $\cdot y r$ fiducial exposure to solar neutrinos. It yields an interaction rate of $49 \pm 3_{\text {stat }} \pm 4_{\text {syst }}$ counts/(day·100 ton) [12].

\section{Sudbury Neutrino Observatory}

The Sudbury Neutrino Observatory (SNO) was a 1,000 ton heavy-water Čerenkov detector [13] situated $2 \mathrm{~km}$ underground in INCO's Creighton mine in Canada. Another 7,000 tons of ultra-pure light water was used for support and shielding. The heavy water was in an acrylic vessel (12 m diameter and $5 \mathrm{~cm}$ thick) viewed by 9,456 PMT mounted on a geodesic structure $18 \mathrm{~m}$ in diameter; all contained within a polyurethane-coated barrel-shaped cavity ( $22 \mathrm{~m}$ diameter by $34 \mathrm{~m}$ high). The SNO detector has been filled with water in May 1999 and data taking ended in 2006. The solar-neutrino detectors in operation prior to SNO were mainly sensitive to the electron neutrino type; while the use of heavy water by SNO allowed the flux of all three neutrino types to be measured. Neutrinos from ${ }^{8} B$ decay in the Sun were observed in SNO from Čerenkov processes following three reactions: (i) the charged-current (CC) reaction, specific to electron neutrinos $d+v_{e} \rightarrow p+p+e^{-}$. This reaction has a $\mathrm{Q}$ value of $1.4 \mathrm{MeV}$ and the electron energy is strongly correlated with the neutrino energy, providing potential sensitivity to spectral distortions; (ii) the neutral-current $(\mathrm{NC})$ reaction, equally sensitive to all non-sterile neutrino types $(x=e, \mu, \tau)$ 
$v_{x}+d \rightarrow n+p+v_{x}$. This reaction has a threshold of $2.2 \mathrm{MeV}$ and is observed through the detection of neutrons by three different techniques in separate phases of the experiment; and (iii) the elastic-scattering (ES) reaction $v_{x}+e^{-} \rightarrow v_{x}+e^{-}$. This reaction has a substantially lower cross section than the other two and as mentioned before is predominantly sensitive to electron neutrinos.

The relations $\Phi_{\mathrm{CC}}=\phi_{e}, \Phi_{\mathrm{ES}}=\phi_{e}+0.15 \phi_{\mu \tau}$ and $\Phi_{\mathrm{NC}}=\phi_{e}+\phi_{\mu \tau}$ gave SNO the status of an appearance experiment. The SNO experimental plan called for three phases wherein different techniques were employed for the detection of neutrons from the NC reaction. During the first phase, with pure heavy water, neutrons were observed through the Čerenkov light produced when neutrons were captured on deuterium, producing $6.25 \mathrm{MeV}$ gammas. For the second phase, about 2 tons of $\mathrm{NaCl}$ was added to the heavy water and neutron detection was enhanced through capture on $\mathrm{Cl}$, with about $8.6 \mathrm{MeV}$ gamma energy release. For the third phase, the salt was removed and an array of ${ }^{3} \mathrm{He}$-filled proportional counters was installed to provide direct detection of neutrons.

SNO reported results from a joint analysis of Phase I and Phase II data [14]. The effective electron kinetic energy threshold used is $3.5 \mathrm{MeV}$, the lowest analysis threshold yet achieved with water Cherenkov detector data. Overall, the low threshold increased the statistics of the CC and ES events by roughly $30 \%$, and of NC events by $\sim 70 \%$. In units of $10^{6} \mathrm{~cm}^{-2} \mathrm{~s}^{-1}$, a fit in which the free parameters directly describe the total ${ }^{8} B$ neutrino flux and the energy-dependent $v_{e}$ survival probability provides a measure of the total ${ }^{8} B$ neutrino flux $\Phi_{8_{B}}=5.046_{-0.152}^{+0.159+0.107}$. The uncertainty on $\Phi_{8_{B}}$ have been significantly reduced compare to previously published results by SNO. The fit for the survival probability assumes unitarity of the neutrino mixing matrix, and that the underlying neutrino spectrum follows a smoothly-distorted ${ }^{8} B$ shape. The day survival probability extracted by $\mathrm{SNO}$ is parameterized as a second-order polynomial $P_{e e}^{\text {day }}\left(E_{v}\right)=$ $c_{0}+c_{1}\left(E_{v}-10 \mathrm{MeV}\right)+c_{2}\left(E_{v}-10 \mathrm{MeV}\right)^{2}$; while allowing for a linear energy-dependent asymmetry between day and night spectra $A\left(E_{v}\right)=a_{0}+a_{1}\left(E_{v}-10 \mathrm{MeV}\right)$. The clear deviation from unity of the constant term of the day survival probability $c_{0}=0.3435_{-0.0197}^{+0.0205}+0.00066-0.0059$ provides a undeniable signature of solar neutrino oscillations [14]. On the other hand, no evidence for either a significant spectral distortion or a day/night asymmetry was found.

SNO also used data of Phase III to measure the rate of NC interactions in heavy water and precisely determined the total active $\left(v_{x}\right)^{8} B$ solar neutrino flux [15]. The total solar neutrino flux is found to be $5.54_{-0.31}^{+0.33}{ }_{-0.34}^{+0.36} \times 10^{6} \mathrm{~cm}^{-2} \mathrm{~s}^{-1}$, in agreement with Phase I and II measurements and the SSM.

\section{Global Fits}

Constraints on neutrino mixing parameters can be derived by comparing neutrino oscillation SSM predictions with experimental data. A three-flavor, active neutrino oscillation model has four parameters: $\theta_{12}$ and $\theta_{13}$, which quantify the strength of the mixing between flavor and mass eigenstates, and $\Delta m_{21}^{2}$ and $\Delta m_{31}^{2}$, the differences between the squares of the masses of the neutrino propagation eigenstates. Note that the remaining mixing angle, $\theta_{23}$, and the $\mathrm{CP}$-violating phase, $\delta$, are irrelevant for the oscillation analysis of solar neutrino data. The value of $\Delta m_{31}^{2}$ was fixed to $+2.3 \times 10^{-3} \mathrm{eV}^{2}$.

For each set of parameters, the oscillation model was used to predict the rates in the Chlorine [4], Gallium [7] and Borexino [12] experiments, the Super-Kamiokande Phase I zenith spec- 
tra [9] and Phase II day/night spectra [10], SNO Phases I and II survival probability day/night curves [14], and the SNO Phase III rates [15]. The expected rates and spectra were divided by the respective predictions, calculated without oscillations, to remove the effects of the model scaling factors. The unitless rates were then used in a global $\chi^{2}$ calculation. For completeness, the rates and spectrum measured by the antineutrino reactor experiment KamLAND [16] are added in the global fit to constrain even further the solar neutrino mixing parameters. The KamLAND rates and spectrum were predicted using three-flavor vacuum oscillations.

Fig. 11(a) shows an overlay of the global solar and the KamLAND allowed regions in $\tan ^{2} \theta_{12}$ and $\Delta m_{21}^{2}$ parameter space, under a two-flavor hypothesis. Fig. 11(b) shows the same overlay for the three-flavor hypothesis that allows the value of $\sin ^{2} \theta_{13}$ to be non-zero. The three-flavor contours show the effect of marginalizing both $\Phi_{8_{\mathrm{B}}}$ and $\sin ^{2} \theta_{13}$ at each point in space.
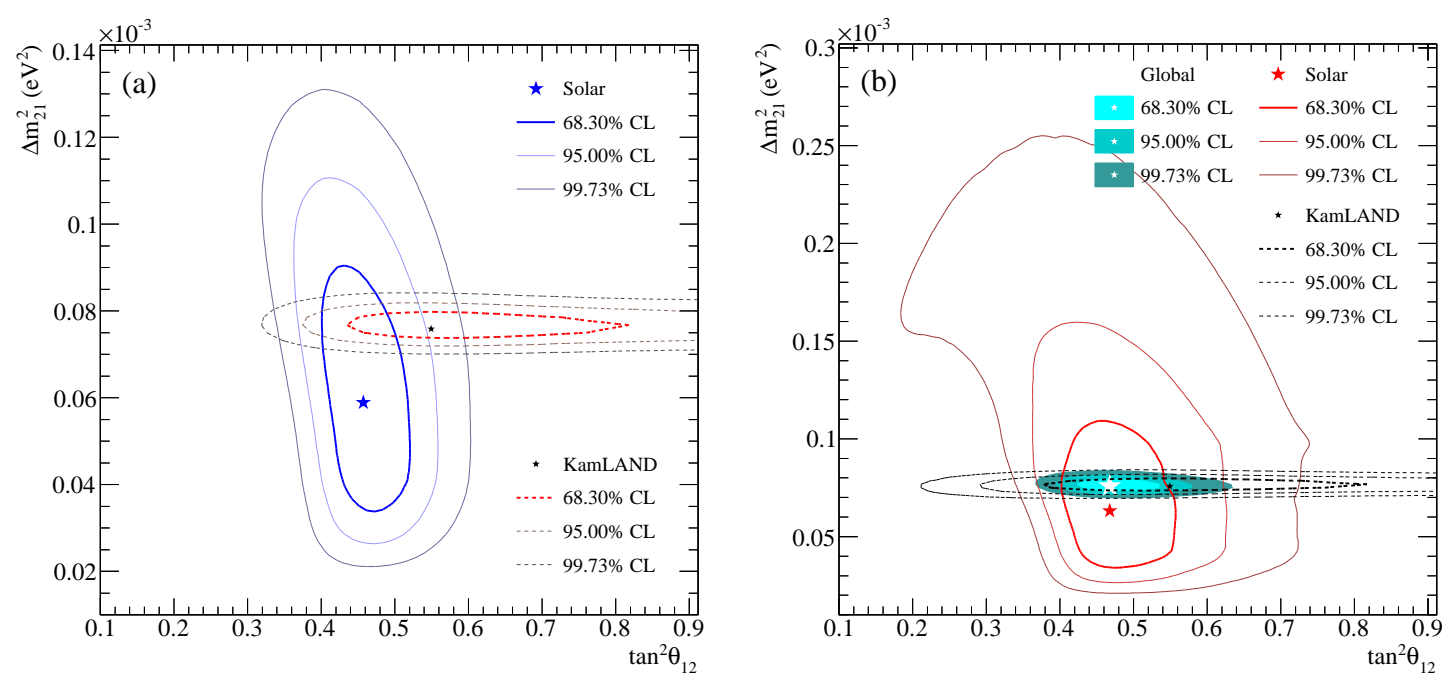

Figure 1: Solar and KamLAND oscillation parameter analysis for a) a two-flavor oscillation hypothesis and b) a three-flavor hypothesis. The solar data includes Cl, SAGE, Gallex/GNO, Borexino, SK-I zenith and SK-II day/night spectra, SNO Phase I+II survival probability day/night curves and SNO Phase III integral rates. The $\chi^{2}$ is minimized with respect to all undisplayed parameters, including $\sin ^{2} \theta_{13}$ and $\Phi_{8_{B}}[14$.

Table 1 summarizes the oscillation parameters from a two-flavor oscillation analysis, while Table 2 summarizes the results from a three-flavor oscillation analysis, performed in the context of a global fit [14].

\section{News and Near Future}

The new Borexino measurement [17] of $v$-e elastic scattering from ${ }^{8} B$ solar neutrinos with a $3 \mathrm{MeV}$ energy threshold is not included in the global fit of the previous section. This result is limited by statistics, but it is nevertheless relevant because it is probing low energy ${ }^{8} B$ neutrinos. The rate of solar neutrino-induced electron scattering events above this energy is $0.217 \pm 0.038 \pm$ 0.008 counts/(day 100 ton), which corresponds to $\Phi_{8_{B}}^{\mathrm{ES}}=2.4 \pm 0.4 \pm 0.1 \times 10^{6} \mathrm{~cm}^{-2} \mathrm{~s}^{-1}$, in good agreement with measurements from SNO and SuperKamiokande. 


\begin{tabular}{lcc}
\hline \hline Oscillation analysis & $\tan ^{2} \theta_{12}$ & $\Delta m_{21}^{2}\left(\mathrm{eV}^{2}\right)$ \\
\hline Solar & $0.457_{-0.041}^{+0.038}$ & $5.89_{-2.16}^{+2.13} \times 10^{-5}$ \\
Solar+KamLAND & $0.457_{-0.029}^{+0.040}$ & $7.59_{-0.21}^{+0.20} \times 10^{-5}$ \\
\hline & $\chi_{\min }^{2} / \mathrm{ndf}$ & $\Phi_{8_{\mathrm{B}}}\left(\times 10^{6} \mathrm{~cm}^{-2} \mathrm{~s}^{-1}\right)$ \\
\hline Solar & $67.5 / 89$ & $5.104_{-0.148}^{+0.199}$ \\
Solar+KamLAND & $82.8 / 106$ & $5.013_{-0.148}^{+0.119}$ \\
\hline \hline
\end{tabular}

Table 1: Best-fit neutrino oscillation parameters and extracted ${ }^{8} \mathrm{~B}$ flux from a two-flavor oscillation analysis. Uncertainties listed are $\pm 1 \sigma$ after the $\chi^{2}$ was minimized with respect to all other parameters.

\begin{tabular}{lcc}
\hline \hline Oscillation analysis & $\tan ^{2} \theta_{12}$ & $\Delta m_{21}^{2}\left(\mathrm{eV}^{2}\right)$ \\
\hline Solar & $0.468_{-0.050}^{+0.052}$ & $6.31_{-2.58}^{+2.49} \times 10^{-5}$ \\
Solar+KamLAND & $0.468_{-0.033}^{+0.042}$ & $7.59_{-0.21}^{+0.21} \times 10^{-5}$ \\
\hline & $\chi_{\min }^{2} / \mathrm{ndf}$ & $\Phi_{8_{\mathrm{B}}}\left(\times 10^{6} \mathrm{~cm}^{-2} \mathrm{~s}^{-1}\right)$ \\
\hline Solar & $67.4 / 89$ & $5.115_{-0.193}^{+0.159}$ \\
Solar+KamLAND & $81.4 / 106$ & $5.087_{-0.159}^{+0.171}$ \\
\hline \multicolumn{2}{c}{$\sin ^{2} \theta_{13}\left(\times 10^{-2}\right)$} \\
\hline Solar & $<8.10(95 \%$ C.L. $)$ \\
Solar+KamLAND & \multicolumn{2}{c}{$2.00_{-1.63}^{+2.09}$} \\
\hline \hline
\end{tabular}

Table 2: Best-fit neutrino oscillation parameters and extracted ${ }^{8} \mathrm{~B}$ flux from a three-flavor oscillation analysis. Uncertainties listed are $\pm 1 \sigma$ after the $\chi^{2}$ was minimized with respect to all other parameters.

Preliminary solar neutrino results of the third phase of the SuperKamiokande presented at this conference [18] have already been submitted for publication [19]. With improved detector calibrations, a full detector simulation, and improved analysis methods, the systematic uncertainty on the total neutrino flux has been reduced to $\pm 2.1 \%$. This leads to a significant contraction of the systematic uncertainty compared to the first phase. The energy threshold of ${ }^{8} B$ neutrino events of SK-III is also pushed lower with respect to SK-I. It yields an ES rate of $2.32 \pm 0.04 \pm 0.05$ $\times 10^{6} \mathrm{~cm}^{-2} \mathrm{sec}^{-1}$, in agreement with previous measurements.

It is clear that the uncertainty on the solar mixing angle $\theta_{12}$ has been noticeably reduced with data analyses that use lower energy thresholds since it enhanced the experimental ability to explore the SSM prediction of energy-dependent matter-induced neutrino oscillation. Very soon, Borexino and SNO will release new results on ${ }^{7} B e$ and ${ }^{8} B$ neutrinos, respectively. Borexino reported at ICHEP2010 a precise day/night asymmetry measurement $A_{\mathrm{DN}}=0.007 \pm 0.073$ [20]. It is foreseen that the statistical precision of Borexino will allow for a detailed investigation of mixing solutions. Expect further improvement with SNO's final joint model-independent fit of the solar survival probability with data from Phases I, II and III.

\section{Summary}

Solar neutrino oscillation is clearly established by the combination of the results from the 
chlorine, gallium, SK, Borexino and SNO experiments. The real-time data of SK, Borexino and SNO do not show large energy distortion nor time-like asymmetry. SNO provided the first direct evidence of flavor conversion of solar electron neutrinos by comparing the $\mathrm{CC}$ and $\mathrm{NC}$ rates. Matter effects seem to explain the energy dependence of solar oscillation, and Large Mixing Angle (LMA) solutions are favored.

After 30 years of hard labor from the nuclear and particle physics community, the Solar Neutrino Problem is now an industry for precise measurements of neutrino oscillation parameters with the next generation of solar neutrino and long baseline neutrino experiments at the horizon.

\section{Acknowledgments}

This article builds upon the careful and detailed work of many people. The author has been financially supported in Canada by the Natural Sciences and Engineering Research Council (NSERC), the Canada Research Chair (CRC) Program, and the Canadian Foundation for Innovation (CFI).

\section{References}

[1] J.N. Bahcall, H.M. Pinsonneault, and S. Basu, Ap. J. 555, 990 (2001).

[2] C. Pena-Garay and A. Serenelli, arXiv:0811.2424 (2008); A. Serenelli, S. Basu, J. Ferguson, and M. Asplund, Ap. J. L., 705, L123 (2009).

[3] R. Davis, Jr., Phys. Rev. Lett., 302 (1964).

[4] B.T. Cleveland et al., Ap. J. 496, 505 (1998).

[5] J.N. Abdurashitov et al., Phys. Rev. C 60, 055801 (1999).

[6] W. Hampel et al., Phys. Lett. B 447, 127 (1999); M. Altmann et al., Phys. Lett. B 490, 16 (2000).

[7] J.N. Abdurashitov et al., Phys. Rev. C 80015807 (2009).

[8] Y. Suzuki et al., Nucl. Phys. (Proc. Suppl.) 34, 54 (1995).

[9] J. Hosaka et al., Phys. Rev. D 73, 112001 (2006).

[10] J. P. Cravens et al., Phys. Rev. D 78032002 (2008).

[11] G. Alimonti et al., Nucl. Inst. Meth. A 600, 568 (2009).

[12] C. Arpesella et al., Phys. Rev. Lett. 101, 091302, (2008).

[13] J. Boger et al., Nucl. Inst. Meth. A 449, 172 (2000).

[14] B. Aharmim et al., Phys. Rev. C 81, 055504 (2010).

[15] B. Aharmim et al., Phys. Rev. Lett. 101, 111301 (2008).

[16] S. Abe et al., Phys. Rev. Lett. 100, 221803 (2008).

[17] G. Bellini et al., Phys. Rev. D 82, 033006 (2010).

[18] Hiroyuki Sekiya in these proceedings.

[19] S. Abe et al., submitted to PRD, arXiv:1010.0118 (2010).

[20] Sandra Zavatarelli in these proceedings. 